\title{
Higgs alignment and the top quark
}

\author{
Estia J. Eichten ${ }^{1, *}$ and Kenneth Lane ${ }^{2, \dagger}$ \\ ${ }^{1}$ Theoretical Physics Group, Fermi National Accelerator Laboratory, \\ P.O. Box 500, Batavia, Illinois 60510, USA \\ ${ }^{2}$ Department of Physics, Boston University, \\ 590 Commonwealth Avenue, Boston, Massachusetts 02215, USA
}

(Received 8 March 2021; accepted 17 May 2021; published 14 June 2021)

\begin{abstract}
There is a surprising connection between the top quark and Higgs alignment in Gildener-Weinberg multi-Higgs doublet models. Were it not for the top quark and its large mass, the coupling of the $125 \mathrm{GeV}$ Higgs boson $H$ to gauge bosons and fermions would be indistinguishable from those of the Standard Model Higgs. The top quark's coupling to a single Higgs doublet breaks this perfect alignment in higher orders of the Coleman-Weinberg loop expansion of the effective potential. But the effect is still small, $\lesssim \mathcal{O}(1 \%)$, and probably experimentally inaccessible.
\end{abstract}

DOI: 10.1103/PhysRevD.103.115022

\section{INTRODUCTION}

The $125 \mathrm{GeV}$ Higgs boson $H$ discovered at the LHC in 2012 [1,2] is consistent in all measurements with the single Higgs boson of the Standard Model (SM). This is dramatically illustrated in Fig. 1 where the couplings of fermions and weak gauge bosons to $H$ as measured by ATLAS and CMS are plotted. This degree of agreement is puzzling. Many well-motivated attempts to cure the problems of the SM-most famously, naturalness-require two or more Higgs multiplets. Why, then, does $H$ have SM couplings? The usual answer is "Higgs alignment" [3-6]. However, with a few exceptions that rely on elaborate global symmetries or supersymmetry [7-10], implementations of alignment suffer large radiative corrections.

In Gildener-Weinberg (GW) multi-Higgs models of electroweak (EW) symmetry breaking [13], the classical Lagrangian is scale-invariant—so that the Higgs potential is purely quartic and fermions acquire mass only from Higgs boson vacuum expectation values. At tree level, $H$ is a Goldstone boson of spontaneously broken scale symmetry. And, in this approximation, $H$ naturally has the same structure as the Goldstone bosons eaten by $W^{ \pm}$and $Z^{0}$. In $N$-Higgs-doublet models (NHDMs),

\footnotetext{
*eichten@fnal.gov

†lane@bu.edu
}

Published by the American Physical Society under the terms of the Creative Commons Attribution 4.0 International license. Further distribution of this work must maintain attribution to the author(s) and the published article's title, journal citation, and DOI. Funded by SCOAP .

$$
\Phi_{i}=\frac{1}{\sqrt{2}}\left(\begin{array}{c}
\sqrt{2} \phi_{i}^{+} \\
\rho_{i}+i a_{i}
\end{array}\right), \quad i=1,2, \ldots N
$$

these Goldstone bosons are

$$
w^{ \pm}=\sum_{i=1}^{N} v_{i} \phi_{i}^{ \pm} / v, \quad z=\sum_{i=1}^{N} v_{i} a_{i} / v,
$$

where $v_{i}$ is the VEV of the $C P$-even scalar $\rho_{i}$ and $v=\sqrt{\sum_{i=1}^{N} v_{i}^{2}}$. The Higgs boson is

$$
H=\sum_{i=1}^{N} v_{i} \rho_{i} / v
$$

Thus, $H$ has exactly the same couplings to EW gauge bosons and to fermions (and, hence, to the gluon and the photon) as the single Higgs boson of the Standard Model; i.e., $H$ is aligned.

In Sec. II of this paper, we show that, but for the top quark, this alignment would be perfect through second order in the Coleman-Weinberg loop expansion of the effective potential [14]. The top quark's presence upsets perfect alignment, but only by a small amount, at most $\mathcal{O}(1 \%)$. In Sec. III we discuss the experimental consequences of this alignment. In short, experimental searches for new Higgs bosons, such as $H^{\prime}, A$, and $H^{ \pm}$, via weak vector boson fusion or decay and Drell-Yan production in association with $H$, will remain fruitless. We also update the promising paths to discovery of these new Higgses at the LHC. They rely on the fact that these new bosons must lie below 400-500 GeV. 

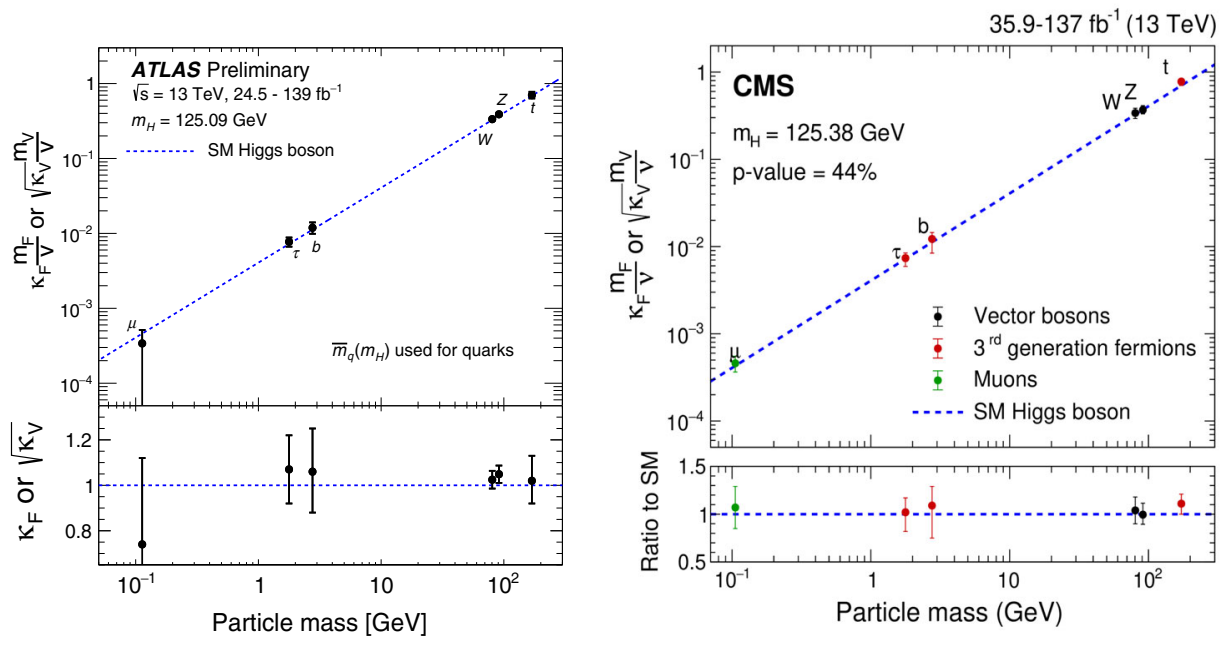

FIG. 1. The mass-dependent couplings of quarks, leptons and the $W$ and $Z$ as measured by ATLAS ([11]) and CMS ([12]).

\section{HIGGS ALIGNMENT IN THE GW-2HDM}

We discuss the top quark's role in Higgs alignment in the context of an $N=2$ Higgs doublet model introduced by Lee and Pilaftsis in 2012 [15]. However, by the GlashowWeinberg criterion that all quarks of a given electric charge must couple to a single Higgs doublet to avoid flavorchanging neutral current interactions mediated by neutral Higgs exchange [16], our conclusion is true in any GWNHDM. This GW-2HDM was updated in 2018 [17] to make it consistent with LHC data at the time. The modification used the following $\mathbb{Z}_{2}$ symmetry on the Higgs doublets and fermions:

$$
\begin{array}{rlrl}
\Phi_{1} & \rightarrow-\Phi_{1}, & \Phi_{2} & \rightarrow \Phi_{2}, \quad \psi_{L} \rightarrow-\psi_{L}, \\
\psi_{u R} & \rightarrow \psi_{u R}, \quad \psi_{d R} & \rightarrow \psi_{d R} .
\end{array}
$$

This is the usual type-I 2HDM [18], but with $\Phi_{1}$ and $\Phi_{2}$ interchanged. (The effect of this is that experimental lower limits on $\tan \beta=v_{2} / v_{1}$ in other type-I models are lower limits on $\cot \beta$ in this model.) The most important experimental constraint came from CMS [19] and ATLAS [20] searches for charged Higgs decay into $t \bar{b}$. Consistency with those experiments required $\tan \beta \lesssim 0.5$ for $M_{H^{ \pm}} \lesssim 500 \mathrm{GeV}$. This is discussed in Sec. III.

The GW tree-level potential for this model is purely quartic [13] so that, since all masses in the model arise from Higgs VEVs, the Lagrangian is scale-invariant at this level:

$$
\begin{aligned}
V_{0}\left(\Phi_{1}, \Phi_{2}\right)= & \lambda_{1}\left(\Phi_{1}^{\dagger} \Phi_{1}\right)^{2}+\lambda_{2}\left(\Phi_{2}^{\dagger} \Phi_{2}\right)^{2} \\
& +\lambda_{3}\left(\Phi_{1}^{\dagger} \Phi_{1}\right)\left(\Phi_{2}^{\dagger} \Phi_{2}\right)+\lambda_{4}\left(\Phi_{1}^{\dagger} \Phi_{2}\right)\left(\Phi_{2}^{\dagger} \Phi_{1}\right) \\
& +\frac{1}{2} \lambda_{5}\left(\left(\Phi_{1}^{\dagger} \Phi_{2}\right)^{2}+\left(\Phi_{2}^{\dagger} \Phi_{1}\right)^{2}\right) .
\end{aligned}
$$

The five quartic couplings $\lambda_{i}$ in Eq. (5) are real and, so, $V_{0}$ is $C P$-invariant. The couplings $\lambda_{1,2}>0$ for positivity of the potential.
The trivial minimum of $V_{0}$ occurs at $\Phi_{1}=\Phi_{2}=0$. But a nontrivial flat minimum of $V_{0}$ can occur on the ray

$$
\Phi_{1 \beta}=\frac{1}{\sqrt{2}}\left(\begin{array}{c}
0 \\
\phi c_{\beta}
\end{array}\right), \quad \Phi_{2 \beta}=\frac{1}{\sqrt{2}}\left(\begin{array}{c}
0 \\
\phi s_{\beta}
\end{array}\right)
$$

where $c_{\beta}=\cos \beta, s_{\beta}=\sin \beta$ with $\beta \neq 0, \pi / 2$ a fixed angle and $0<\phi<\infty$ a real mass scale. ${ }^{1}$ The nontrivial extremal conditions are

$$
\lambda_{1}+\frac{1}{2} \lambda_{345} \tan ^{2} \beta=\lambda_{2}+\frac{1}{2} \lambda_{345} \cot ^{2} \beta=0,
$$

where $\lambda_{345}=\lambda_{3}+\lambda_{4}+\lambda_{5}<0$ for positivity of $V_{0}$. Equations (7) hold in all orders of the loop expansion of the effective potential [13]; this is important in our subsequent development [see Eqs. (12), (13), (14)]. This extremum spontaneously (but not explicitly) breaks scale invariance, as well as the EW gauge symmetry, and $H$ is the corresponding Goldstone boson.

Following Ref. [21], we use the "aligned basis" of the Higgs fields because the scalars' mass matrices will remain very nearly diagonal in that basis beyond the tree approximation (also see Ref. [6]). That is the essence of Higgs alignment in GW models and, in this and similar models, it is broken, but only slightly, by the top quark. This basis is

$$
\begin{gathered}
\Phi=\Phi_{1} c_{\beta}+\Phi_{2} s_{\beta}=\frac{1}{\sqrt{2}}\left(\begin{array}{c}
\sqrt{2} w^{+} \\
H+i z
\end{array}\right), \\
\Phi^{\prime}=-\Phi_{1} s_{\beta}+\Phi_{2} c_{\beta}=\frac{1}{\sqrt{2}}\left(\begin{array}{c}
\sqrt{2} H^{+} \\
H^{\prime}+i A
\end{array}\right) .
\end{gathered}
$$

\footnotetext{
${ }^{1}$ It is easily proved that any such purely quartic potential as well as its first derivatives vanish at any extremum so that $V_{0}\left(\Phi_{i \beta}\right)=0[21]$.
} 
On the ray Eq. (6) on which $V_{0}$ has nontrivial extrema, these fields are $\Phi=(0, \phi) / \sqrt{2}$ and $\Phi^{\prime}=0$. In this basis, $H, z, w^{ \pm}$are massless and unmixed with the $H^{\prime}, A, H^{ \pm}$ whose "masses" are

$M_{H^{\prime}}^{2}=-\lambda_{345} \phi^{2}, \quad M_{A}^{2}=-\lambda_{5} \phi^{2}, \quad M_{H^{ \pm}}^{2}=-\frac{1}{2} \lambda_{45} \phi^{2}$.

Thus, the flat potential is indeed a minimum on the ray (6) (albeit degenerate with the trivial one) if, like $\lambda_{345}, \lambda_{5}$ and $\lambda_{45}=\lambda_{4}+\lambda_{5}$ are negative.

To establish the top quark's role in Higgs alignment of GW-NHDMs, it suffices to consider this model in one-loop order of the effective potential, $V_{0}+V_{1}$. This potential provides a lower minimum than $V_{0}=0$ by picking out a particular value $v$ of $\phi$, explicitly breaking the scale symmetry of $V_{0}$, and giving $H$ a nonzero mass. The one-loop potential is [22]

$$
V_{1}=\frac{1}{64 \pi^{2}} \sum_{n} \alpha_{n} \bar{M}_{n}^{4}\left(\ln \frac{\bar{M}_{n}^{2}}{\Lambda_{\mathrm{GW}}^{2}}-k_{n}\right)
$$

Only very massive particles contribute to $V_{1}$. They are $n=\left(W^{ \pm}, Z, t, H^{\prime}, A, H^{ \pm}\right)$in this model. The constants are $\alpha_{n}=(6,3,-12,1,1,2) ; k_{n}=5 / 6$ for the weak gauge bosons and $3 / 2$ for scalars and the top-quark. ${ }^{2}$ The background-field-dependent masses $\bar{M}_{n}^{2}$ in Eq. (10) are $[15,23]$

$$
\bar{M}_{n}^{2}=\left\{\begin{array}{c}
M_{n}^{2}\left(2\left(\Phi^{\dagger} \Phi+\Phi^{\prime \dagger} \Phi^{\prime}\right) / \phi^{2}\right)=M_{n}^{2}\left(H^{2}+H^{\prime 2}+\cdots\right) / \phi^{2}, \quad n \neq t \\
M_{t}^{2}\left(2 \Phi_{1}^{\dagger} \Phi_{1} /\left(\phi c_{\beta}\right)^{2}\right)=M_{t}^{2}\left(\left(H c_{\beta}-H^{\prime} s_{\beta}\right)^{2}+\cdots\right) /\left(\phi c_{\beta}\right)^{2}, \\
=M_{t}^{2}\left(\left(H-H^{\prime} \tan \beta\right)^{2}+\cdots\right) / \phi^{2}
\end{array}\right.
$$

where $M_{n}^{2} \propto \phi^{2}$ is the actual squared "mass" of particle $n$. The form of $\bar{M}_{t}^{2}$ is dictated by the type-I coupling of fermions to the $\Phi_{1}$ doublet in Eq. (4). This difference controls the breaking of Higgs alignment through second order in the loop expansion. The renormalization scale $\Lambda_{\mathrm{GW}}$ will be fixed relative to the Higgs VEV $v=246 \mathrm{GeV}$ in Eq. (15) below.

The one-loop extremal conditions are [13]

$\left.\frac{\partial\left(V_{0}+V_{1}\right)}{\partial H}\right|_{\langle\rangle+\delta_{1} H+\delta_{1} H^{\prime}}=\left.\frac{\partial\left(V_{0}+V_{1}\right)}{\partial H^{\prime}}\right|_{\langle\rangle+\delta_{1} H+\delta_{1} H^{\prime}}=0$.

Here, we follow GW's analysis by expanding around the tree-level VEVs $\langle H\rangle=\phi,\left\langle H^{\prime}\right\rangle=0$ while allowing for $\mathcal{O}\left(V_{1}\right)$ shifts $\delta_{1} H$ and $\delta_{1} H^{\prime}$ in those VEVs-and from perfect Higgs alignment. Recall that the tree-level extremal conditions $\left(\partial V_{0} / \partial H\right)_{\langle\rangle}=\left(\partial V_{0} / \partial H^{\prime}\right)_{\langle\rangle}=0$ remain in force. To $\mathcal{O}\left(V_{1}\right)$ this expansion results in

$\left.\frac{\partial V_{1}}{\partial H}\right|_{\langle\rangle}=\frac{1}{16 \pi^{2} v} \sum_{n} \alpha_{n} M_{n}^{4}\left(\ln \frac{M_{n}^{2}}{\Lambda_{\mathrm{GW}}^{2}}+\frac{1}{2}-k_{n}\right)=0$,

\footnotetext{
${ }^{2} V_{1}$ was calculated in the Landau gauge using the $\overline{\mathrm{MS}}$ renormalization scheme.
}

$$
\begin{aligned}
& \left.\frac{\partial^{2} V_{0}}{\partial H^{\prime 2}}\right|_{\langle\rangle} \delta_{1} H^{\prime}+\left.\frac{\partial V_{1}}{\partial H^{\prime}}\right|_{\langle\rangle} \\
& \quad=M_{H^{\prime}}^{2} \delta_{1} H^{\prime}-\frac{\alpha_{t} M_{t}^{4} \tan \beta}{16 \pi^{2} v}\left(\ln \frac{M_{t}^{2}}{\Lambda_{\mathrm{GW}}^{2}}+\frac{1}{2}-k_{t}\right)=0,
\end{aligned}
$$

where, by Eq. (11), the first derivative with respect to $H^{\prime}$ of the $n \neq t$ terms in $V_{1}$ vanish because they are quadratic in $H^{\prime}$. Equation (13) provides a definition of the renormalization scale $\Lambda_{\mathrm{GW}}$ in terms of the VEV $\phi=v$ at which the minimum of $V_{1}$ occurs. It can be rewritten as [13]

$$
\begin{aligned}
0 & =\sum_{n} \alpha_{n} M_{n}^{4}\left(\ln \frac{M_{n}^{2}}{\Lambda_{\mathrm{GW}}^{2}}+\frac{1}{2}-k_{n}\right) \\
& =A+\frac{1}{2} B+B \ln \left(\frac{v^{2}}{\Lambda_{\mathrm{GW}}^{2}}\right)
\end{aligned}
$$

where $A=\sum_{n} \alpha_{n} M_{n}^{4}\left(\ln \left(M_{n}^{2} / v^{2}\right)-k_{n}\right)$ and $B=\sum_{n} \alpha_{n} M_{n}^{4}$, so that $\ln \left(\Lambda_{\mathrm{GW}}^{2} / v^{2}\right)=A / B+\frac{1}{2}{ }^{3}$. Note that $M_{n}^{2} \propto v^{2}$ so that $\Lambda_{\mathrm{GW}} / v$ is a function of coupling constants only.

From Eq. (14), the shift $\delta_{1} H^{\prime}$ in $\left\langle H^{\prime}\right\rangle$ is given by the tadpole formula:

\footnotetext{
${ }^{3}$ As discussed in Ref. [13], Eq. (13) does not lead to a minimum of $V_{1}$ unless $B>0$. With the known masses of $W^{ \pm}, Z, t$ and $H, B>0$; see Eq. (17).
} 
$\delta_{1} H^{\prime}=-\left.\frac{1}{M_{H^{\prime}}^{2}} \frac{\partial V_{1}}{\partial H^{\prime}}\right|_{\langle\rangle}=\frac{\alpha_{t} M_{t}^{4} \tan \beta}{16 \pi^{2} M_{H^{\prime}}^{2} v}\left(\ln \frac{M_{t}^{2}}{\Lambda_{\mathrm{GW}}^{2}}+\frac{1}{2}-k_{t}\right)$.

As an example of its magnitude, we take $M_{H^{\prime}}=400 \mathrm{GeV}$, $\Lambda_{\mathrm{GW}}=260 \mathrm{GeV}$ and $\tan \beta=0.5$. Then $\delta_{1} H^{\prime}=1.57 \mathrm{GeV}$ which, when added in quadrature with $v=246 \mathrm{GeV}$, amounts to an increase of $0.002 \%{ }^{4}$

Equation (16) establishes the connection of the top quark to Higgs alignment: The large mass of the top quark ensures its appearance in the effective potential $V_{1}$ while the Glashow-Weinberg criterion [16] implies $\left(\partial \bar{M}_{t}^{2} / \partial H^{\prime}\right)_{\langle\rangle} \neq 0$; hence the small $\mathcal{O}\left(V_{1}\right)$ shift away from perfect alignment. The elements of the $C P$-even mass matrix $\mathcal{M}_{0^{+}}^{2}$ in $\mathcal{O}\left(V_{1}\right)$ further emphasize this connection:

$$
\begin{gathered}
\mathcal{M}_{H H}^{2}=\left.\frac{\partial^{2} V_{1}}{\partial H^{2}}\right|_{\langle\rangle}=\frac{1}{8 \pi^{2} v^{2}} \sum_{n} \alpha_{n} M_{n}^{4}, \\
\mathcal{M}_{H H^{\prime}}^{2}=\left.\frac{\partial^{3} V_{0}}{\partial H \partial H^{\prime 2}}\right|_{\langle\rangle} \delta_{1} H^{\prime}+\left.\frac{\partial^{2} V_{1}}{\partial H \partial H^{\prime}}\right|_{\langle\rangle} \\
=-\frac{\alpha_{t} M_{t}^{4} \tan \beta}{16 \pi^{2} v^{2}}\left(\ln \frac{M_{t}^{2}}{\Lambda_{\mathrm{GW}}^{2}}+\frac{5}{2}-k_{t}\right), \\
\mathcal{M}_{H^{\prime} H^{\prime}}^{2}=\left.\frac{\partial^{2} V_{0}}{\partial H^{\prime 2}}\right|_{\langle\rangle}+\left.\frac{\partial^{3} V_{0}}{\partial H^{\prime 3}}\right|_{\langle\rangle} \delta_{1} H^{\prime}+\left.\frac{\partial^{2} V_{1}}{\partial H^{\prime 2}}\right|_{\langle\rangle} \\
=M_{H^{\prime}}^{2}+\frac{\alpha_{t} M_{t}^{4}}{8 \pi^{2} v^{2}}\left(\ln \frac{M_{t}^{2}}{\Lambda_{\mathrm{GW}}^{2}}+\frac{1}{2}-k_{t}+\tan ^{2} \beta\right) .
\end{gathered}
$$

At this level, only the top quark prevents $\mathcal{M}_{0^{+}}^{2}$ being diagonal and the Higgs boson being completely aligned.
To repeat: Because the Glashow-Weinberg criterion applies to any EW model in which quarks of a given charge acquire all their mass from the scalars, the top quark's role in Higgs alignment holds in any GW-NHDM. The additional complications of the two-loop effective potential do not alter this conclusion. ${ }^{5}$

\section{EXPERIMENTAL CONSEQUENCES}

ATLAS and CMS discovered the $125 \mathrm{GeV}$ Higgs $H$ relatively easily because of its rather strong coupling to weak bosons: production via $W W$ and $Z Z$ fusion and decay to $W W^{*}$ and $Z Z^{*}$. It is worth remembering that, despite its lower statistics, $H \rightarrow Z Z^{*} \rightarrow 4$ leptons was much more convincing at first than $H \rightarrow \gamma \gamma$. Furthermore, $g g$ fusion of $H$ via the top-quark loop was important because the $\bar{t} t H$ coupling has its full-strength value, $M_{t} / v$. Because of this success, it seems, a lamp-post strategy has been adopted for many searches of beyond-Standard-Model (BSM) Higgs bosons. This is especially true for heavier neutral Higgses such as $H^{\prime}$ and $A$. The web abounds with searches for $H^{\prime}$ and $A$ production via weak-boson fusion or gluon fusion followed by their decay to weak boson pairs or to $Z H$, as well as $W^{ \pm} Z \rightarrow H^{ \pm} \rightarrow W^{ \pm} Z$ or $W^{ \pm} H$; see, e.g., Refs. [24] and [25].

All of these processes are strongly hindered by Higgs alignment in GW models. ${ }^{6}$ This fact is codified in the (nonGoldstone) Higgs bosons' interactions with electroweak bosons and fermions. They are taken from Ref. [17] but, because the $H-H^{\prime}$ mixing angle $\delta \lesssim \mathcal{O}(1 \%)$ [21], so that the rates for Higgs-alignment-violating processes are suppressed by at least a factor of $10^{-4}$, it is more illuminating to write them in terms of the aligned-basis fields $H$ and $H^{\prime}$ rather than the mass eigenstates $H_{1}=H \cos \delta-H^{\prime} \sin \delta$ and $H_{2}=H \sin \delta+H^{\prime} \cos \delta$.

$$
\begin{aligned}
\mathcal{L}_{\mathrm{EW}}= & i e H^{-} \stackrel{\leftrightarrow}{\partial_{\mu}} H^{+}\left(A^{\mu}+Z^{\mu} \cot 2 \theta_{W}\right)+\frac{e}{\sin 2 \theta_{W}}\left(H^{\prime} \stackrel{\leftrightarrow}{\partial_{\mu}} A\right) Z^{\mu}+\frac{i g}{2}\left(H^{+} \stackrel{\leftrightarrow}{\partial_{\mu}}\left(H^{\prime}+i A\right) W^{-\mu}-H^{-} \stackrel{\leftrightarrow}{\partial_{\mu}}\left(H^{\prime}-i A\right) W^{+\mu}\right) \\
& +H\left(g M_{W} W^{+\mu} W_{\mu}^{-}+\frac{1}{2} \sqrt{g^{2}+g^{\prime 2}} M_{Z} Z^{\mu} Z_{\mu}\right)+\left(H^{2}+H^{\prime 2}+A^{2}\right)\left(\frac{1}{4} g^{2} W^{+\mu} W_{\mu}^{-}+\frac{1}{8}\left(g^{2}+g^{\prime 2}\right) Z^{\mu} Z_{\mu}\right) \\
& +H^{+} H^{-}\left(e^{2}\left(A_{\mu}+Z_{\mu} \cot 2 \theta_{W}\right)^{2}+\frac{1}{4} g^{2} W_{\mu}^{+} W^{-\mu}\right) .
\end{aligned}
$$

\footnotetext{
${ }^{4}$ Because $\delta_{1} H$ is not determined in $\mathcal{O}\left(V_{1}\right)$, we can set it to zero here. This is consistent with our expectation that $\delta H=\mathcal{O}\left(\delta^{2}\right)$ where $\delta=\mathcal{O}\left(V_{1}\right)$ is the $H-H^{\prime}$ mixing angle.

${ }^{5}$ E. J. Eichten and K. Lane, in preparation.

${ }^{6}$ Similarly suppressed are the production in future lepton colliders of BSM scalars singly-produced in $e^{+} e^{-}, \mu^{+} \mu^{-} \rightarrow Z \rightarrow Z H^{\prime} / A$ or via weak-boson fusion.
} 


$$
\begin{aligned}
\mathcal{L}_{Y}= & \frac{\sqrt{2} \tan \beta}{v} \sum_{k, l=1}^{3}\left[H^{+}\left(\bar{u}_{k L} V_{k l} m_{d_{l}} d_{l R}-\bar{u}_{k R} m_{u_{k}} V_{k l} d_{l L}+m_{\ell_{k}} \bar{\nu}_{k L} \ell_{k R} \delta_{k l}\right)+\text { H.c. }\right] \\
& -\left(\frac{v+H-H^{\prime} \tan \beta}{v}\right) \sum_{k=1}^{3}\left(m_{u_{k}} \bar{u}_{k} u_{k}+m_{d_{k}} \bar{d}_{k} d_{k}+m_{\ell_{k}} \bar{\ell}_{k} \ell_{k}\right)-\frac{i A \tan \beta}{v} \sum_{k=1}^{3}\left(m_{u_{k}} \bar{u}_{k} \gamma_{5} u_{k}-m_{d_{k}} \bar{d}_{k} \gamma_{5} d_{k}-m_{\ell_{k}} \bar{\ell}_{k} \gamma_{5} \ell_{k}\right),
\end{aligned}
$$

where $V=U_{L}^{\dagger} D_{L}$ is the CKM matrix. Note that gluon fusion and two-photon decay of $H^{\prime}$ and $A$ via a top-quark loop are suppressed by $\tan ^{2} \beta<0.25$ [17]. So long ${\operatorname{as~} \tan ^{2} \beta}^{2}$ is not much smaller, this can, in principle, be overcome by the data expected in Run 3. And, so long as most BSM Higgs decays are to fermion pairs, the $\tan ^{2} \beta$ suppression does not affect decay branching ratios.

The prospects for testing the GW-2HDM (and similar models) are much brighter than these comments suggest. ${ }^{7}$ They stem from the fact that the one-loop approximation for the Higgs boson's mass in Eq. (17) implies a sum rule for the BSM Higgs masses [15,17,26,27]:

$$
\left(M_{H^{\prime}}^{4}+M_{A}^{4}+2 M_{H^{ \pm}}^{4}\right)^{1 / 4}=540 \mathrm{GeV} .
$$

Equation (22) tells us that the BSM scalars are lighter than 400-500 GeV. ${ }^{8}$ In using this sum rule, we shall assume that $M_{A}=M_{H^{ \pm}}$, an assumption justified by the fact that it makes the BSM scalars' contribution to the $T$-parameter vanish identically $[28,29]$.)

The principal search modes for the BSM scalars are via gluon fusion:

$$
\begin{gathered}
g g \rightarrow H^{+} \bar{t} b \quad \text { with } H^{+} \rightarrow t \bar{b} \text { and } W^{+} H^{\prime} ; \\
\quad g g \rightarrow A \rightarrow \bar{b} b, \quad \bar{t} t \quad \text { and } Z H^{\prime} ; \\
g g \rightarrow H^{\prime} \rightarrow \bar{b} b, \quad \bar{t} t \quad \text { and } Z A, W^{ \pm} H^{\mp} .
\end{gathered}
$$

Cross sections for these processes (with the $\tan \beta$ dependence factored out) are in Fig. 2. It is unlikely that an $H^{\prime}$ or $A \rightarrow \bar{b} b$ lighter than $2 M_{t}$ can be seen above the QCD background [30] unless it is accompanied by $Z \rightarrow \ell^{+} \ell^{-}$. If it is, then the $\bar{b} b \ell^{+} \ell^{-}$signal should be with reach of ATLAS and CMS capabilities. The decays $H^{ \pm} \rightarrow W^{ \pm} H^{\prime}$ and $A\left(H^{\prime}\right) \rightarrow Z H^{\prime}(A)$ are quickly dominant once the channels open because the weak boson becomes longitudinally polarized and the decay rate proportional to

\footnotetext{
${ }^{7}$ The following discussion is updated from Ref. [21] to include analyses using the full LHC Run 2 data set of $139 \mathrm{fb}^{-1}$.

${ }^{8}$ Because of limits from LHC searches for charged Higgses lighter than $M_{t}$ and neutral Higgses lighter than $M_{H}$ (see the ATLAS and CMS websites noted above), it is likely that they are heavier than $\sim 180 \mathrm{GeV}$.
}

$p^{3} / M_{W, Z}^{2}$. Thus, they are important near the upper end of the mass range allowed by the sum rule (22).

(1) $g g \rightarrow H^{+} \bar{t} b \rightarrow \bar{t} t \bar{b} b$

There have been four searches for this process relevant to the mass range of the GW-2HDM $[19,20,31,32]$. The first of these was a CMS search at $8 \mathrm{TeV}$; the other three are from $13-\mathrm{TeV}$ data with the last an ATLAS search using the full Run 2 data set of $139 \mathrm{fb}^{-1}$. The $8-\mathrm{TeV}$ search by CMS was used to set the limit $\tan \beta \lesssim 0.5$ for $180 \mathrm{GeV}<M_{H^{ \pm}}<$ $500 \mathrm{GeV}$. Subsequent searches have not improved on this limit despite the larger datasets. For example, the limit for the $200-500 \mathrm{GeV}$ mass range extracted from Ref. [32] is $\tan \beta<1.10 \pm 0.14$. The main reason for this disappointing outcome is the large $\bar{t} t$ background at low masses and the fact that its rate increases with energy faster than the signal does. Given the payoff of a significant improvement in the limit on $\tan \beta$, we urge ATLAS and CMS to find a way to overcome the problems of searches at low masses.

There have been no dedicated searches for $g g \rightarrow H^{+} \bar{t} b$ with $H^{+} \rightarrow W^{+} H^{\prime} \rightarrow W^{+} \bar{b} b$ but this and the main process (without resonant $\bar{b} b$ ) have the same final state. Hence, $H^{+} \rightarrow W^{+} H^{\prime}$ may

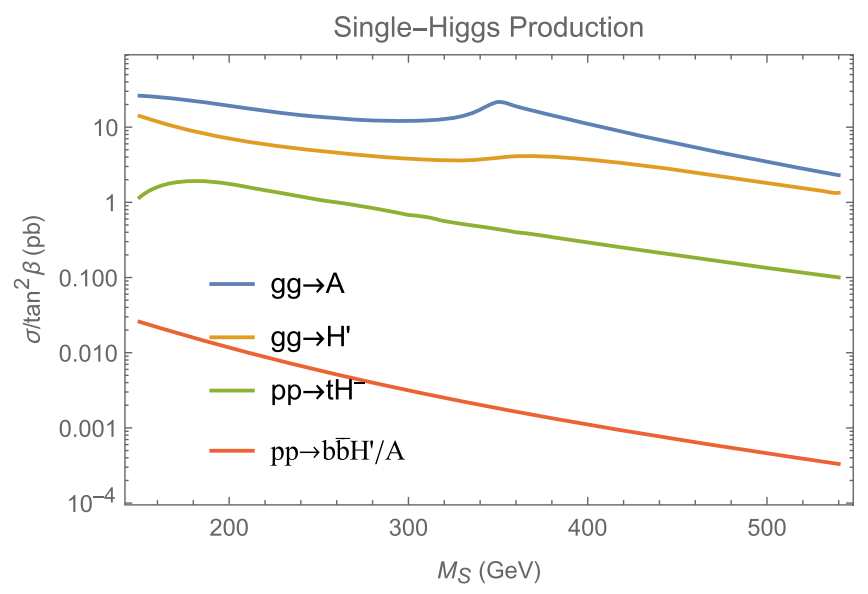

FIG. 2. The cross sections for $\sqrt{s}=13 \mathrm{TeV}$ at the LHC for single Higgs production processes in the alignment limit $(\delta \rightarrow 0)$ of the GW-2HDM with the dependence on $\tan \beta$ scaled out. Both charged Higgs states are included in $p p \rightarrow t \bar{b} H^{-}$. From Ref. [17]. 
unintentionally be included in a search for $H^{+} \rightarrow t \bar{b}$. Even if that happened, the model expectation $\sigma\left(g g \rightarrow H^{+} \bar{t} b\right)=0.075 \mathrm{pb}$ for $M_{H^{ \pm}} \simeq 400 \mathrm{GeV}$ and $\tan \beta=0.5$ is well below the new $95 \% \mathrm{CL}$ ATLAS limit of $0.42 \mathrm{pb}$.

(2) $g g \rightarrow A / H^{\prime} \rightarrow \bar{t} t$

A search by CMS with $35.9 \mathrm{fb}^{-1}$ of data at $13 \mathrm{TeV}$ for $A / H^{\prime} \rightarrow \bar{t} t$ with low mass, $400<$ $M_{A / H^{\prime}}<750 \mathrm{GeV}$, is in Ref. [33]. CMS presented results in terms of allowed and excluded regions of the "coupling strength" $g_{\varphi}=\lambda_{\varphi t \bar{t}} /\left(M_{t} / v\right)$ and for fixed width-to-mass ratio $\Gamma_{\varphi} / M_{\varphi}=0.5-25 \%$. In the GW-2HDM, $g_{\varphi}=\tan \beta$. For the $C P$-odd case, $\varphi=A$, with $400 \mathrm{GeV}<M_{A}<500 \mathrm{GeV}$ and all $\Gamma_{A} / M_{A}$ considered, the region $\tan \beta<0.5$ is not excluded. ${ }^{9}$ This is possibly due to an excess at $400 \mathrm{GeV}$ that corresponds to a global (local) significance of $1.9(3.5 \pm 0.3) \sigma$ for $\Gamma_{A} / M_{A} \simeq 4 \%$. The CMS paper notes that higher-order electroweak corrections to the SM $t \bar{t}$ threshold production may account for the excess and that further improvement in the theoretical description is needed.

(3) $g g \rightarrow A\left(H^{\prime}\right) \rightarrow Z H^{\prime}(A) \rightarrow \ell^{+} \ell^{-} \bar{b} b$

There have been three published searches so far: [34-36]. The latter ATLAS search updates the former one with the full Run 2 dataset. As noted above, the decay rates of these processes are proportional to $p^{3}$ and, therefore, they are sensitive to the available phase space. They quickly become dominant when $M_{A}=M_{H^{ \pm}} \gtrsim 400 \mathrm{GeV}$ or $M_{H}^{\prime} \gtrsim$ $350 \mathrm{GeV}[21,37]$. Two examples of this are shown in Table I which has been updated from Ref. [21] to include the recent ATLAS analysis. The full Run 2 dataset has significantly improved the ATLAS limits on $\sigma B$ which were $255 \mathrm{fb}$ and $105 \mathrm{fb}$ for a $36.1 \mathrm{fb}^{-1}$ dataset in Ref. [34]; in particular, the GW-2HDM

\footnotetext{
${ }^{9}$ The same appears to be true for $\varphi=H^{\prime}$ with $\Gamma_{H^{\prime}} / M_{H^{\prime}} \gtrsim 1 \%$.
}

TABLE I. $\quad 95 \% \mathrm{CL}$ upper limits on $\sigma\left(p p \rightarrow A\left(H^{\prime}\right)\right) B\left(A\left(H^{\prime}\right) \rightarrow\right.$ $\left.Z H^{\prime}(A)\right) B\left(H^{\prime}(A) \rightarrow \bar{b} b\right)$ via gluon fusion from ATLAS [36], CMS [35] and GW-2HDM calculations for two cases of large $M_{A}$ or $M_{H^{\prime}}$. The CMS limits include $B\left(Z \rightarrow e^{+} e^{-}, \mu^{+} \mu^{-}\right)$; the ATLAS limits and GW-2HDM predictions do not. Masses are in $\mathrm{GeV}$ and $\sigma B$ in femtobarns. $M_{A}=M_{H^{ \pm}}$is assumed and $M_{H^{\prime}}$ is taken from Eq. (22). Model cross sections are from Fig. 2 with $\tan \beta=0.50$.

\begin{tabular}{lcccc}
\hline \hline$M_{A}=M_{H^{ \pm}}$ & $M_{H^{\prime}}$ & ATLAS & CMS & GW-2HDM \\
\hline 400 & 300 & 90 & 75 & 65 \\
300 & 500 & 51 & 50 & 100 \\
\hline \hline
\end{tabular}

mass point $M_{A}=M_{H^{ \pm}}=300 \mathrm{GeV}, M_{H^{\prime}}=500 \mathrm{GeV}$ is now excluded for $\tan \beta=0.5$; it would be allowed for $\tan \beta<0.35$.

A search for $A\left(H^{\prime}\right) \rightarrow Z H^{\prime}(A) \rightarrow \ell^{+} \ell^{-} \bar{b} b$ using Contur [38] was carried out at the Les Houches 2019 "Physics at TeV colliders" workshop [37]. It showed no significant sensitivity to these processes for $\tan \beta<0.5$ except near $M_{A} \geq 400 \mathrm{GeV}$ where there was a $\geq 2 \sigma$ exclusion requiring $\tan \beta \lesssim 0.3$. This exclusion was based on ATLAS $8-\mathrm{TeV}$ data for $\ell^{+} \ell^{-}+$jets. This study was recently extended using several full Run 2 ATLAS datasets (see Ref. [39]). The $1 \sigma$ exclusion now covers $\tan \beta<0.9$ extending down to $\tan \beta \simeq 0.43$ at $M_{A}=150-200 \mathrm{GeV}$ and $\tan \beta \gtrsim 0.23$ at $M_{A} \gtrsim 400 \mathrm{GeV}$. The greatest sensitivity came from ATLAS jet and top measurements except near $M_{A} \geq 400 \mathrm{GeV}$ where ATLAS 4-lepton measurements dominated.

\section{ACKNOWLEDGMENTS}

We are grateful for valuable guidance from Stephen Martin and for our collaboration with Eric Pilon. We have benefited from discussions on the LHC data with Tulika Bose, Kevin Black, Gustav Brooijmans, Jon Butterworth, Viviana Cavaliere, William Murray, and David Sperka.
[1] G. Aad et al. (ATLAS Collaboration), Observation of a new particle in the search for the Standard Model Higgs boson with the ATLAS detector at the LHC, Phys. Lett. B 716, 1 (2012).

[2] S. Chatrchyan et al. (CMS Collaboration), Observation of a new boson at a mass of $125 \mathrm{GeV}$ with the CMS experiment at the LHC, Phys. Lett. B 716, 30 (2012).

[3] F. Boudjema and A. Semenov, Measurements of the SUSY Higgs selfcouplings and the reconstruction of the Higgs potential, Phys. Rev. D 66, 095007 (2002).
[4] J. F. Gunion and H. E. Haber, The $C P$ conserving two Higgs doublet model: The approach to the decoupling limit, Phys. Rev. D 67, 075019 (2003).

[5] M. Carena, I. Low, N. R. Shah, and C.E. M. Wagner, Impersonating the standard model Higgs boson: Alignment without decoupling, J. High Energy Phys. 04 (2014) 015.

[6] H. E. Haber, Approximate Higgs alignment without decoupling, in 53rd Rencontres de Moriond on QCD and High Energy Interactions (2018), pp. 139-142, https://cds.cern $. c h /$ record/2301199? ln=en. 
[7] I. P. Ivanov, Minkowski space structure of the Higgs potential in 2HDM. II. Minima, symmetries, and topology, Phys. Rev. D 77, 015017 (2008).

[8] P. S. Bhupal Dev and A. Pilaftsis, Maximally symmetric two Higgs doublet model with natural standard model alignment, J. High Energy Phys. 12 (2014) 024; Erratum, 11 (2015) 147.

[9] K. Benakli, M. D. Goodsell, and S. L. Williamson, Higgs alignment from extended supersymmetry, Eur. Phys. J. C 78, 658 (2018).

[10] N. Darvishi and A. Pilaftsis, Quartic coupling unification in the maximally symmetric 2HDM, Phys. Rev. D 99, 115014 (2019).

[11] https://atlas.web.cern.ch/Atlas/GROUPS/PHYSICS/ CombinedSummaryPlots/HIGGS/.

[12] https://cms-results.web.cern.ch/cms-results/public-results/ publications/HIG/index.html.

[13] E. Gildener and S. Weinberg, Symmetry breaking and scalar bosons, Phys. Rev. D 13, 3333 (1976).

[14] S. R. Coleman and E. J. Weinberg, Radiative corrections as the origin of spontaneous symmetry breaking, Phys. Rev. D 7, 1888 (1973).

[15] J.S. Lee and A. Pilaftsis, Radiative corrections to scalar masses and mixing in a scale invariant two Higgs doublet model, Phys. Rev. D 86, 035004 (2012).

[16] S. L. Glashow and S. Weinberg, Natural conservation laws for neutral currents, Phys. Rev. D 15, 1958 (1977).

[17] K. Lane and W. Shepherd, Natural stabilization of the Higgs bosons mass and alignment, Phys. Rev. D 99, 055015 (2019).

[18] G. C. Branco, P. M. Ferreira, L. Lavoura, M. N. Rebelo, M. Sher, and J. P. Silva, Theory and phenomenology of twoHiggs-doublet models, Phys. Rep. 516, 1 (2012).

[19] V. Khachatryan et al. (CMS Collaboration), Search for a charged Higgs boson in pp collisions at $\sqrt{s}=8 \mathrm{TeV}$, J. High Energy Phys. 11 (2015) 018.

[20] M. Aaboud et al. (ATLAS Collaboration), Search for charged Higgs bosons decaying into top and bottom quarks at $\sqrt{s}=13 \mathrm{TeV}$ with the ATLAS detector, J. High Energy Phys. 11 (2018) 085.

[21] K. Lane and E. Pilon, Phenomenology of the new light Higgs bosons in Gildener-Weinberg models, Phys. Rev. D 101, 055032 (2020).

[22] S. P. Martin, Two loop effective potential for a general renormalizable theory and softly broken supersymmetry, Phys. Rev. D 65, 116003 (2002).

[23] R. Jackiw, Functional evaluation of the effective potential, Phys. Rev. D 9, 1686 (1974).

[24] https://twiki.cern.ch/twiki/bin/view/AtlasPublic.

[25] https://cms-results.web.cern.ch/cms-results/public-results/ publications/HIG/SUS.html.

[26] K. Hashino, S. Kanemura, and Y. Orikasa, Discriminative phenomenological features of scale invariant models for electroweak symmetry breaking, Phys. Lett. B 752, 217 (2016).

[27] J. Braathen, S. Kanemura, and M. Shimoda, Two-loop analysis of classically scale-invariant models with extended Higgs sectors, J. High Energy Phys. 03 (2021) 297.

[28] R. A. Battye, G. D. Brawn, and A. Pilaftsis, Vacuum topology of the two Higgs doublet model, J. High Energy Phys. 08 (2011) 020.

[29] A. Pilaftsis, On the classification of accidental symmetries of the two Higgs doublet model potential, Phys. Lett. B 706, 465 (2012).

[30] A. M. Sirunyan et al. (CMS Collaboration), Search for lowmass resonances decaying into bottom quark-antiquark pairs in proton-proton collisions at $\sqrt{s}=13 \mathrm{TeV}$, Phys. Rev. D 99, 012005 (2019).

[31] A. M. Sirunyan et al. (CMS Collaboration), Search for a charged Higgs boson decaying into top and bottom quarks in events with electrons or muons in proton-proton collisions at $\sqrt{\mathrm{s}}=13 \mathrm{TeV}$, J. High Energy Phys. 01 (2020) 096.

[32] G. Aad et al. (ATLAS Collaboration), Search for charged Higgs bosons decaying into a top quark and a bottom quark at $\sqrt{s}=13 \mathrm{TeV}$ with the ATLAS detector, arXiv: 2102.10076 .

[33] A. M. Sirunyan et al. (CMS Collaboration), Search for heavy Higgs bosons decaying to a top quark pair in protonproton collisions at $\sqrt{s}=13 \mathrm{TeV}$, J. High Energy Phys. 04 (2020) 171.

[34] M. Aaboud et al. (ATLAS Collaboration), Search for a heavy Higgs boson decaying into a $Z$ boson and another heavy Higgs boson in the $\ell \ell b b$ final state in $p p$ collisions at $\sqrt{s}=13 \mathrm{TeV}$ with the ATLAS detector, Phys. Lett. B 783, 392 (2018).

[35] A. M. Sirunyan et al. (CMS Collaboration), Search for new neutral Higgs bosons through the $\mathrm{H} \rightarrow \mathrm{ZA} \rightarrow \ell^{+} \ell^{-} \mathrm{b} \overline{\mathrm{b}}$ process in pp collisions at $\sqrt{s}=13 \mathrm{TeV}$, J. High Energy Phys. 03 (2020) 055.

[36] G. Aad et al. (ATLAS Collaboration), Search for a heavy Higgs boson decaying into a $\mathrm{Z}$ boson and another heavy Higgs boson in the $\ell \ell b b$ and $\ell \ell W W$ final states in $p p$ collisions at $\sqrt{s}=13 \mathrm{TeV}$ with the ATLAS detector, Eur. Phys. J. C 81, 396 (2021).

[37] G. Brooijmans et al., Les Houches 2019 physics at $\mathrm{TeV}$ colliders: New physics working group report, arXiv:2002 .12220 .

[38] J. M. Butterworth, D. Grellscheid, M. Krämer, B. Sarrazin, and D. Yallup, Constraining new physics with collider measurements of Standard Model signatures, J. High Energy Phys. 03 (2017) 078.

[39] https://hepcedar.gitlab.io/contur-webpage/results/G-W/index .html. 